\title{
Influence d'apports de matière organique sur la culture de mil et d'arachide sur un sol sableux du Nord-Sénégal. I. - Bilans de consommation, production et développement racinaire
}

\author{
Limamoulaye CISSE \& Georges VACHAUD* \\ Institut Sénégalais de Recherches agricoles, CNRA - BAMBEY - Sénégal \\ * C.N.R.S. - Institut de Mécanique de Grenoble, B.P. 68, F 38402 Saint-Martin-d'Hères Cedex
}

RÉSUMÉ

\begin{abstract}
Dans le cas d'une expérimentation de longue durée menée sur un sol dégradé du Nord-Sénégal, on s'est attaché à caractériser l'influence de l'apport de matière organique sur les bilans de consommation et de production pour une rotation mil-arachide.

Un suivi détaillé des transferts hydriques, basé sur l'utilisation couplée d'humidimétrie neutronique et de tensiométrie, après sélection des sites de mesure pour leur représentativité spatiale, a mis en évidence des différences considérables de consommation en eau, de pertes en drainage et de production végétale entre le traitement et le témoin. Une caractérisation des propriétés hydrodynamiques a montré que l'effet de l'apport en matière organique sur les propriétés de transfert et de rétention hydrique du sol n'est pas significatif. Cet apport induit de forts contrastes au niveau du développement racinaire donc une bien meilleure alimentation hydrique (et minérale comme on le verra dans un article suivant), et une production bien supérieure. Il apparaît enfin nettement que la consommation totale en eau peut expliquer assez bien la production de l'arachide. Pour le mil, et pour les conditions extrêmes rencontrées dans cette expérimentation, il semble par contre que ce seul paramètre n'ait pas un pouvoir explicatif suffisant.
\end{abstract}

Mots clés additionnels : Amendement organique, sol dégradé, production végétale, bilan hydrique, caractérisation hydrodynamique, variabilité spatiale.

Effect of organic amendment on cultivation of millet and groundnut in a degraded sandy soil in North Senegal. I. Water consumption, root development and crop production.

Within the framework of long-term agronomic research on the conditions of degraded soils in North Senegal, an experiment was done to determine the effect of organic amendment on water consumption and crop production of millet and peanut in rotation. A detailed analysis of soil water transfer was done, with simultaneous use of neutron moisture meter and tensiometer at measurement sites selected for their spatial representativity. Very significant differences were obtained in terms of water consumption, drainage losses and crop production between treatment and control plots. In a first stage, it was shown that organic amendment had no effect on water transfer and water-holding capacity of soil. However, very high contrasts in term of root development were obtained between treatment and control. It is believed that this factor was responsible for the greater efficiency of water and nutrient absorption, and therefore for the higher production of the crop cultivated on the treatment. Finally, it appeared that total water consumption was, for groundnut, a fairly good estimator of total production whereas for millet this variable could not be used as a sensitive parameter in a predictive model of production.

Additional key words : Hydrodynamic characterization, spatial variability.

\section{INTRODUCTION}

Le Centre-Nord du Sénégal qui comprend les régions de Louga, de Thies et de Diourbel est une zone de cultures pluviales dont les principales sont l'arachide (sur
$500000 \mathrm{ha}$ ) et le mil souna (sur $400000 \mathrm{ha}$ ). Ces surfaces représentent près de 45 p. 100 des superficies totales cultivées par an au Sénégal en arachide, mil et sorghe.

Cette zone est caractérisée par l'existence de 2 sai- 
sons très contrastées : une longue saison sèche puis une saison des pluies de juillet à septembre, avec une pluviométrie moyenne (période 1970-1986) voisine de $350 \mathrm{~mm} / \mathrm{an}$. C'est donc une zone à pluviométrie généralement faible et souvent limitante pour les cultures, et dont la variabilité interannuelle est en outre très élevée.

Les sols cultivés dominants sont des sols ferrugineux tropicaux peu lessivés (environ 80 p. 100 des sols cultivés) très sableux. Ils ont été décrits par de nombreux auteurs et notamment par BONFILS \& FAURE (1959). Ces sols se sont développés sur du sable quaternaire d'origine fluviale et marine qui a subi par la suite des actions éoliennes. Ils présentent un profil très homogène de la surface jusqu'à plus de $4 \mathrm{~m}$ de profondeur. Leur fraction argileuse composée essentiellement de kaolinite est très faible ( 2 à $3 \mathrm{p}$. 100); leur horizon humifère, très peu différencié, présente des taux de matière organique de 0,2 à 0,5 p. 100. Ces sols ont des capacités d'échange cationique très faibles ( 1 à $3 \mathrm{meq} / 100 \mathrm{~g}$ de sol). Du fait de leur texture essentiellement sableuse, de leur composition minéralogique et de leur pauvreté en matière organique, ils ont un faible pouvoir tampon.

La pression démographique qui s'est accentuée vers les années 1960 a entraîné, dans cette zone très anciennement cultivée, un bouleversement des systèmes de culture. La culture itinérante, caractérisée par des jachères de durée plus ou moins longue qui permettaient aux sols de ne pas trop se dégrader, s'est transformée progressivement en une culture fixée avec une utilisation continue des sols.

Il est clair que l'absence de restitution des résidus de récoltes, l'insuffisance des apports d'engrais minéraux qui ne pouvaient pas compenser les exportations minérales ont entraîné une baisse du statut organique des sols et des réserves en bases échangeables, en particulier celles en Ca, Mg et K (PIERI, 1976). Ainsi la fertilité de ces sols s'est progressivement dégradée pour aboutir, dans certains cas, à un niveau tel que leur productivité très faible contraignait les paysans à les abandonner. En conséquence la production arachidière dans cette zone est passée en moyenne de 350000 tonnes/an entre 1962-1967 à 290000 tonnes/an entre 1968-1971.

On a été ainsi amené à mettre en place en 1972, à Thilmakha, dans un site représentatif de cette zone, une expérimentation agronomique dont l'objet est de tester des techniques culturales pour la régénération d'un sol sableux dégradé et d'en mesurer les effets sur l'arachide et le mil. Cet article présente certains résultats obtenus depuis 1983 sur ce dispositif expérimental dans le cadre d'une action de recherche soutenue par l'AIEA (Agence Internationale pour l'Energie Atomique). Une présentation détaillée est donnée par ailleurs par CISSE (1986).

\section{PRÉSENTATION DE L’EXPÉRIMENTATION}

\section{A. Dispositif de base et conduite des cultures}

Depuis le départ, le dispositif expérimental est constitué de 2 séries de 6 blocs de Fischer comprenant chacun 8 traitements complètement randomisés sur des parcelles de $90 \mathrm{~m}^{2}$. La distribution des traitements à l'intérieur des blocs est la même dans les 2 séries. Ces traitements concernent plusieurs associations de labour, de fumure minérale, d'apport de fumier, de chaux. Les séries sont cultivées en rotation arachidemil, avec labour réalisé à la traction bovine $(10 \mathrm{~cm}$ de profondeur environ) ; l'apport de fumier (10 p. 100 d'humidité environ) à raison de $10 \mathrm{t} / \mathrm{ha}$, et celui de la chaux $(600 \mathrm{~kg} / \mathrm{ha})$ sont faits uniquement sur la culture d'arachide et leurs effets résiduels sont mesurés sur la culture de mil qui suit.

L'arachide (variété 55-437, 90 jours) est semée au disque avec un écartement entre les lignes de 0,45 m. Au semis, $150 \mathrm{~kg} /$ ha d'engrais ternaire (NPK) de formule 8-18-27 sont apportés. Le mil (SOUNA III, 90 jours) est semé en poquets à la densité de $1 \mathrm{~m} \times 1 \mathrm{~m}$, démariés 10 jours après la levée, à 3 plants. Au semis du mil, $150 \mathrm{~kg} / \mathrm{ha}$ d'engrais ternaire (NPK) de formule 14-7-7 sont apportés. Au démariage et au $41^{\mathrm{e}}$ jour de végétation, $50 \mathrm{~kg} / \mathrm{ha}$ d'urée sont appliqués.

La conduite des cultures (sarclo-binage, protection phytosanitaire, etc...) est correctement assurée en suivant les techniques mises au point par la recherche agronomique.

Les résultats obtenus de 1972 à 1981 au niveau des rendements ont montré que le labour n'augmente pas les rendements de l'arachide ( 7 années sur 10) ou a un faible effet sur ceux-ci. La chaux, seule ou combinée au labour, procure en moyenne des surplus de rendements, par rapport au traitement fertilisation minérale seule, de 40 à 60 p. 100. Les traitements (labour + fumier) et (labour + fumier + chaux) ne diffèrent pas et donnent en moyenne des rendements 2 fois plus élevés que celui du traitement fertilisation minérale. Le fumier apparaît donc comme ayant l'effet le plus important sur les rendements.

Cette analyse nous a amené à choisir les traitements extrêmes (labour + fertilisation minérale) et (labour + fumier + fertilisation minérale), pour être l'objet d'observations et de mesures spécifiques visant à mettre en évidence les effets du fumier sur le sol et la production et en expliquer son action très positive. Ces 2 traitements sont par la suite référencés respectivement par T0 et T1.

L'étude a démarré en 1983 sur une appréciation des effets des 2 traitements sous culture d'arachide. Une caractérisation du sol (y compris les formes d'azote) a été faite sur des échantillons de sol prélevés avant le semis de l'arachide. Sur chacun des 2 traitements choisis on a implanté sur 5 blocs de la série I au centre des parcelles, un tube d'accès pour humidimètre à neutrons à $4 \mathrm{~m}$ de profondeur associé à 6 cellules de prélèvements de solution du sol mises en place autour de chaque tube à $1,5 \mathrm{~m}$ de profondeur pour estimer les pertes minérales par lixiviation sous culture d'arachide (CHOPART, 1980).

L'analyse des résultats obtenus en 1983 a conduit à orienter le protocole de mesures de la façon suivante :

\section{En 1984}

Compte tenu de la faible variabilité spatiale de la texture et des profils hydriques, on a pu réduire à 3 le nombre de répétitions à instrumenter par traitement, ce qui a permis de travailler en même temps sur les 2 cultures et de réserver les répétitions restantes aux interventions et mesures très destructives ou pouvant 
modifier significativement l'effet des traitements étudiés.

L'instrumentation des traitements en tubes d'accès pour humidimètre à neutrons, tensiomètres et cellules de prélèvements de solution du sol est présentée tableau 1. La disposition des cellules a été effectuée pour d'une part comparer la composition de la solution prélevée sous les 2 traitements et, d'autre part, calculer les pertes minérales par lixiviation. Pour le mil, ces pertes sont estimées à la cote $1,80 \mathrm{~m}$, cote maximum atteinte par le système racinaire du mil sur ce type de sol (CHOPART, 1980).

TABLEAU 1

Instrumentation pour mesures de bilans d'eau et de solutés en 1984 et 1985.

Definition of site instrumentation in terms of soil moisture and soil solution concentration measurements.

\begin{tabular}{lcc}
\hline \hline & Arachide & Mil \\
\hline $\begin{array}{l}\text { Nombre de blocs instrumentés } \\
\text { * Mesures neutroniques }\end{array}$ & $3(\times 2)$ & $3(\times 2)$ \\
* Tensiomètres & $0,6-0,9-1,2$ & $0,8-1,1-1,4$ \\
* Cellules de prélèvement & 1,5 et $1,8 \mathrm{~m}$ & 1,7 et $2 \mathrm{~m}$ \\
(solution du sol) & $0,3-0,6-0,9-1,5 \mathrm{~m}$ & $0,3-0,6-0,9-1,8 \mathrm{~m}$ \\
\hline
\end{tabular}

* sur chaque bloc instrumenté.

En outre, un essai de caractérisation des propriétés de transfert et de rétention hydrique du sol a été réalisé après les récoltes sur chacun des 2 traitements afin d'étudier si les apports de matière organique avaient modifié ces propriétés (CISSE \& VACHAUD, 1988a).

Enfin, une caractérisation de l'enracinement du mil dans la couche $0-0,5 \mathrm{~m}$ du sol a été faite par mesure de la masse de racines contenues dans des tranches de sol prélevées dans une fosse de $1 \mathrm{~m}^{2}$ de surface, et relatives aux profondeurs suivantes : $0-0,1 \mathrm{~m} ; 0,1-0,2 \mathrm{~m} ; 0,2$ $0,3 \mathrm{~m}$ et $0,3-0,5 \mathrm{~m}$. Les racines, extraites par lavage sur tamis à maille carrée de $1 \mathrm{~mm}$, sont pesées après $72 \mathrm{~h}$ à l'étuve.

\section{En 1985}

On a poursuivi les mêmes mesures hydriques et tensiométriques et de prélèvement de solution du sol sous les 2 cultures.

L'évolution de l'enracinement du mil et de l'arachide dans les couches $(0-0,1 \mathrm{~m}$ et $0,1 \mathrm{~m}-0,2 \mathrm{~m})$ a été caractérisée par mesure de la densité racinaire aux $15^{\mathrm{e}}$, $30^{\mathrm{e}}, 45^{\mathrm{e}}$ et $75^{\mathrm{e}}$ jour après la levée. A mi-cycle de développement végétatif de l'arachide une caractérisation de l'enracinement de la plante, similaire à celle faite sur le mil en 1984, a été effectuée. Une caractérisation des principaux stades phénologiques ainsi qu'un suivi de l'évolution des teneurs et des mobilisations en azote, phosphore, potassium, calcium et magnésium ont été réalisés sur les 2 plantes (CISSE, 1988). Enfin, le statut organique du sol soumis aux 2 traitements a été étudié par une analyse de la répartition du carbone et de l'azote dans les principales fractions texturales (200$2000,50-200,20-50,2-20$ et $0-2 \mu)$ des horizons $0-0,1 \mathrm{~m}$ et $0,1-0,2 \mathrm{~m}$ du sol.

Seuls les résultats relatifs au bilan hydrique et à la relation consommation en eau-production font l'objet de cette étude.

\section{B. Climat}

La pluviométrie relevée à Thilmakha de 1983 à 1985 est présentée au tableau 2 ainsi que les mesures "d'évaporation bac " obtenue à Bambey. Les années relatives à notre travail se caractérisent, du point de vue pluviométrique, par :

- une saison des pluies très courte en 1983 ( 2 mois) qui ne s'est réellement installée qu'au début du mois d'août et qui a pris fin très précocement. La quantité totale d'eau tombée a été la plus faible de celles relevées depuis le début de l'expérimentation. Deux épisodes de sécheresse ont eu lieu respectivement dans la deuxième et la première décade des mois d'août et de septembre ;

- une date d'installation normale des pluies en 1984, mais une pluviométrie faible présentant une période déficitaire dans la deuxième décade du mois d'août ;

TABLEAU 2

Données climatiques - Pluviométrie décadaire à Thilmakha

Values of rainfall and pan evaporation from 1983 to 1985 (daily values averaged over decads).

\begin{tabular}{|c|c|c|c|c|c|c|c|c|c|c|c|c|c|c|c|c|}
\hline \multirow{2}{*}{ Année } & \multicolumn{3}{|c|}{ Juin } & \multicolumn{3}{|c|}{ Juillet } & \multicolumn{3}{|c|}{ Août } & \multicolumn{3}{|c|}{ Septembre } & \multicolumn{3}{|c|}{ Octobre } & \multirow{2}{*}{$\begin{array}{l}\text { Total } \\
\text { annuel }\end{array}$} \\
\hline & $1^{\mathrm{re}} \mathrm{d}$ & $2^{\mathrm{e}} \mathrm{d}$ & $3^{e} \mathrm{~d}$ & $1^{\mathrm{re}} \mathrm{d}$ & $2^{\mathrm{e}} \mathrm{d}$ & $3^{e} \mathrm{~d}$ & $1^{\mathrm{re}} \mathrm{d}$ & $2^{\mathrm{e}} \mathrm{d}$ & $3^{e} \mathrm{~d}$ & $1^{\mathrm{re}} \mathrm{d}$ & $2^{\mathrm{e}} \mathrm{d}$ & $3^{e} \mathrm{~d}$ & $1^{\mathrm{re}} \mathrm{d}$ & $2^{\mathrm{e}} \mathrm{d}$ & $3^{\mathrm{e}} \mathrm{d}$ & \\
\hline 1983 & 0,0 & 18,0 & 0,0 & 8,6 & 1,0 & 0,0 & 66,8 & 4,0 & 68,2 & 0,0 & 23,5 & 20,5 & 0,0 & 0,0 & 0,0 & 210,6 \\
\hline 1984 & 10,0 & 37,0 & 3,0 & 17,5 & 12,5 & 19,2 & 31,0 & 6,5 & 44,0 & 17,5 & 52,0 & 10,1 & 19,0 & 0,0 & 0,0 & 279,3 \\
\hline 1985 & 0,0 & 0,0 & 28,0 & 0,0 & 20,2 & 20,5 & 11,0 & 82,0 & 91,5 & 33,5 & 51,4 & 1,6 & 7,4 & 0,0 & 0,0 & 347,1 \\
\hline
\end{tabular}

Evaporation bac décadaire en $m m$ mesurée à Bambey

\begin{tabular}{|c|c|c|c|c|c|c|c|c|c|c|c|c|c|c|c|}
\hline \multirow{2}{*}{ Année } & \multicolumn{3}{|c|}{ Juin } & \multicolumn{3}{|c|}{ Juillet } & \multicolumn{3}{|c|}{ Août } & \multicolumn{3}{|c|}{ Septembre } & \multicolumn{3}{|c|}{ Octobre } \\
\hline & $\mathrm{l}^{\text {re }} \mathrm{d}$ & $2^{\mathrm{e}} \mathrm{d}$ & $3^{\mathrm{e}} \mathrm{d}$ & $\mathrm{l}^{\mathrm{rc}} \mathrm{d}$ & $2^{\mathrm{e}} \mathrm{d}$ & $3^{e} \mathrm{~d}$ & $1^{\text {re }} \mathrm{d}$ & $2^{\mathrm{e}} \mathrm{d}$ & $3^{e} d$ & $1^{\text {re }} \mathrm{d}$ & $2^{e} \mathrm{~d}$ & $3^{e} \mathrm{~d}$ & $\mathrm{lre}^{\mathrm{re}}$ & $2^{\mathrm{e}} \mathrm{d}$ & $3^{e} \mathrm{~d}$ \\
\hline 1983 & 90,0 & 86,1 & 79,2 & 75,5 & 82,4 & 88,9 & 76,9 & 72,0 & 59,6 & 66,3 & 62,1 & 56,4 & 62,3 & 75,0 & 73,0 \\
\hline 1984 & 100,4 & 79,7 & 71,3 & 74,1 & 65,1 & 67,6 & 61,1 & 81,2 & 66,7 & 67,3 & 66,3 & 54,2 & 54,9 & 68,3 & 26,2 \\
\hline 1985 & 92,5 & 91,3 & 82,8 & 83,8 & 67,7 & 60,0 & 64,3 & 48,0 & 53,7 & 43,6 & 56,2 & 55,8 & 74,3 & 78,2 & 75,7 \\
\hline
\end{tabular}


- un début de saison des pluies un peu tardif en 1985, avec une pluviométrie annuelle relativement meilleure que celle des 2 années précédentes et caractérisée par des épisodes très pluvieux pendant les 2 dernières décades du mois d'août.

On notera que pour aucune de ces 3 années les besoins en eau du mil et de l'arachide qui s'élèvent, pour les variétés cultivées, à $400-450 \mathrm{~mm}$ dans cette zone (DANCETTE, 1974) n'ont pu être satisfaits.

\section{Sol - Caractéristiques texturales et hydriques}

Les caractéristiques granulométriques du sol sont les suivantes (valeurs en p. 100) :

sable grossier $(>200 \mu) 29,9 \pm 3,3$; sable moyen $(100-200 \mu): 64,1 \pm 3,5$

sable fin $(50-100 \mu): 1,7 \pm 0,3$

et argile + limon $(<50 \mu): 3,9 \pm 0,6$.

Ce sol représente un milieu d'étude remarquablement homogène, comparativement aux expérimentations réalisées précédemment à Bambey par IMBERNON (1982).

Pour soutenir cette observation, on dispose des données suivantes:

- en terme de texture, le taux d'éléments fins (argile + limon) qui a un rôle essentiel au niveau des transferts hydriques reste compris entre 2 et $4,2 \cdot 10^{-2}$ entre 0 et $2 \mathrm{~m}$ de profondeur, avec un écart type spatial voisin de $5 \cdot 10^{-3}$ (valeurs obtenues sur 15 profils à raison d'une mesure tous les $0,1 \mathrm{~m}$ entre 0 et $2 \mathrm{~m}$ ) ;

- en terme de masse volumique, les mesures au cylindre de densité montrent que l'on obtient les

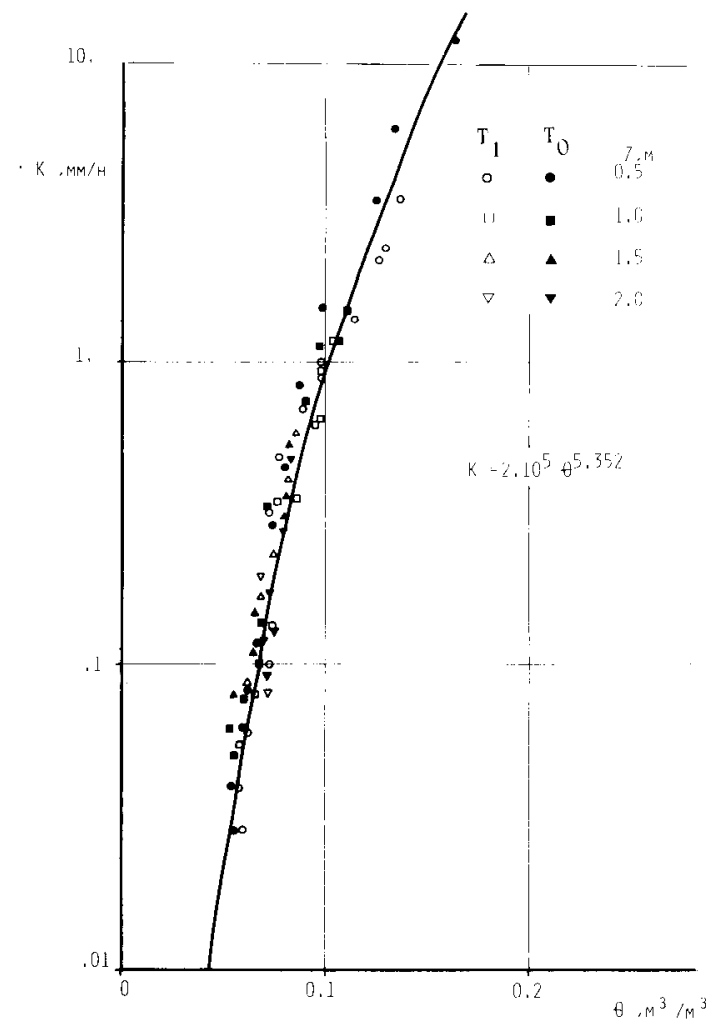

valeurs suivantes :

- pour l'horizon de surface $(0,0,1 \mathrm{~m}): 1,45 \mathrm{~T} / \mathrm{m}^{3}$,

- au-delà (jusqu'à $1,5 \mathrm{~m}$ ) par tranche de $0,1 \mathrm{~m}$ : $1,5 \pm 0,01 \mathrm{~T} / \mathrm{m}^{3}$;

- en terme d'humidité, tous les profils hydriques obtenus par sonde à neutrons en 1983, à raison de 5 répétitions par traitement ne diffèrent en moyenne entre eux à une date donnée que de $5.10^{-3} \mathrm{~m}^{3} / \mathrm{m}^{3}$ par horizon de mesure. Cette valeur étant du même ordre de grandeur que l'incertitude sur la mesure (VAUCLIN et al., 1983), on a pu limiter par la suite à 3 le nombre de répétitions sans pertes d'information significatives (CISSE, 1986) ;

- en terme de paramètres hydrodynamiques (conductivité hydraulique $K(\theta)$ et courbes de rétention $h(\theta)), 2$ essais de caractérisation par infiltration sous charge constante et drainage interne ont été effectués sur les traitements $\mathrm{T} 0$ et $\mathrm{T} 1$. Les résultats détaillés sont analysés par ailleurs (CISSE \& VACHAUD, 1988a). Outre le fait qu'aucun effet dû à l'apport en matière organique n'a pu être observé, on notera :

- qu'une seule courbe de conductivité $K(\theta)$ (fig. 1) peut caractériser tous les points de mesures, profondeurs et traitements confondus. Ce qui nous a incités à utiliser, quand nécessaire, cette courbe pour le calcul des flux par la loi de Darcy,

- que la relation $h(\theta)$ est soumise à une forte hystérésis. Partant toutefois de la même teneur en eau, les valeurs obtenues sur $\mathrm{T} 0$ et $\mathrm{T} 1$ sont identiques.

Enfin du fait de la faible quantité d'éléments fins et de la forte conductivité à «saturation» (environ $200 \mathrm{~mm} / \mathrm{h}$ ), le sol est extrêmement filtrant ; sa réserve en eau est très faible, et de l'ordre de $70 \mathrm{~mm}$, par $\mathrm{m}$ de sol.

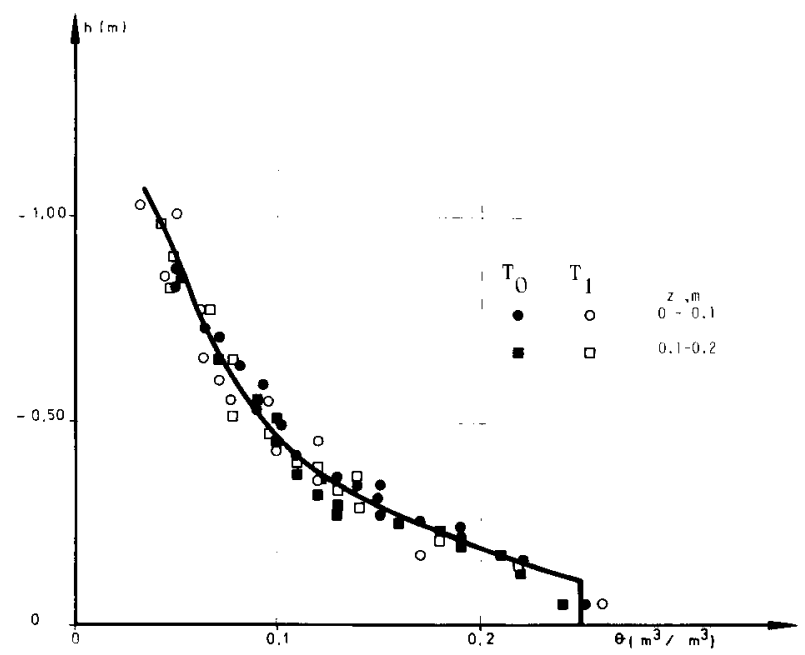

Figure 1 


\section{EFFETS DES TRAITEMENTS SUR LES BILANS HYDRIQUES}

La première information frappante obtenue en 1983 au niveau des mesures de profils hydriques effectuées au cours du cycle végétatif de l'arachide concerne le contraste entre les cinétiques d'humectation du sol sur les traitements T0 et T1. A titre d'exemple on trouvera figures 2 et 3 respectivement les profils hydriques initiaux mesurés avant semis et ceux obtenus à 3 dates caractéristiques du cycle : 15 jours après semis (20/08), maximum de stock hydrique $(13 / 09)$ et une semaine avant récolte $(22 / 10)$, et les courbes d'avancement du

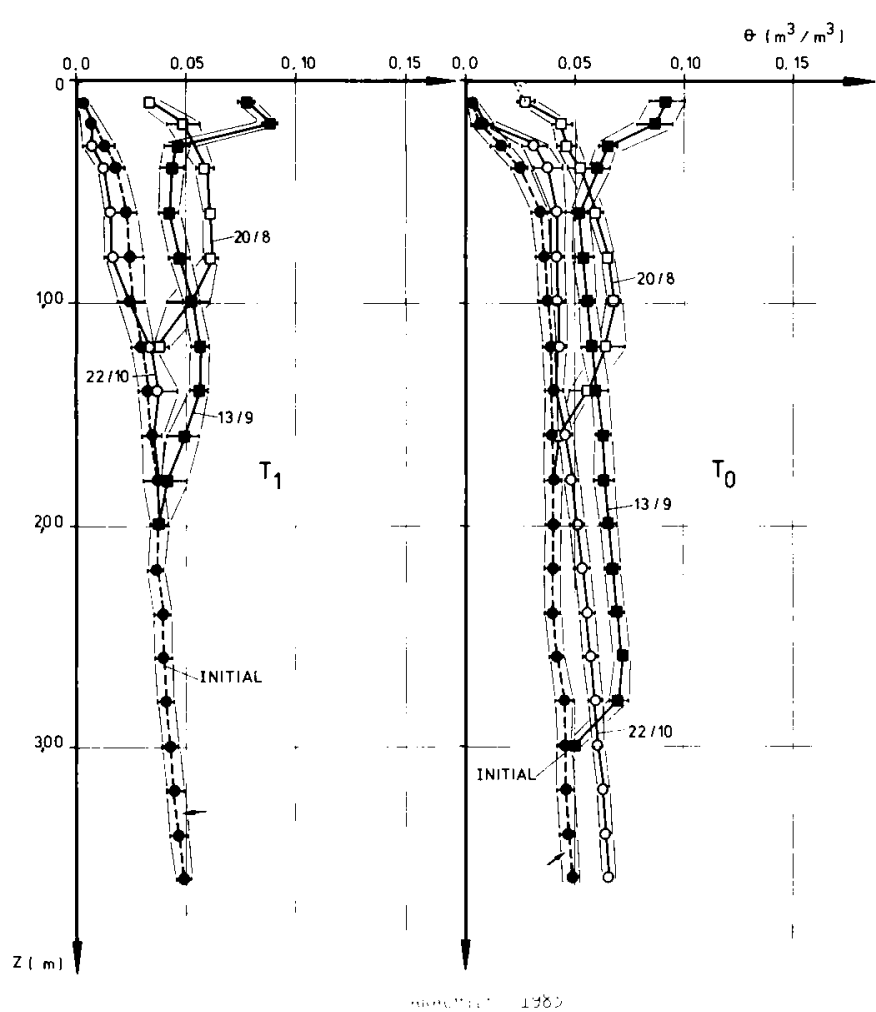

Figure 2

Comparaison entre profils hydriques obtenus à des dates caractéristiques sur les traitements TI et T0, arachide, 1983 (3 répétitions par traitement). ( $\theta$ teneur en eau. $Z$ profondeur en $m$.)

Comparison between water content profiles measured at three dates in 1983 under groundnut for treatments TO and TI 3 replicates for each treatment). ( $\theta$ volumetric water content, $Z$ depth.)

front d'humectation, relatives aux 2 traitements choisis. Sur chacune de ces 2 figures on a représenté les bandes d'incertitudes relatives aux 5 répétitions par traitement. On notera que pour chaque traitement, la dispersion des mesures spatiales est du même ordre de grandeur que l'incertitude de la mesure $\left( \pm 0,05 \mathrm{~m}^{3} / \mathrm{m}^{3}\right)$. On mettra donc à profit cette très grande homogénéité du milieu pour utiliser une relation unique $K(\theta)$ ou $h(\theta)$ pour caractériser le milieu du point de vue hydrodynamique. La validation de cette hypothèse est donnée dans un autre article (CISSE \& VACHAUD, 1988a).

Malgré la très faible pluviométrie, on notera que les percolations passent au-delà de la dernière cote de mesure disponible $(3,70 \mathrm{~m})$ sur le traitement $\mathrm{T} 0$, alors que sur T1 le front d'humectation s'arrête à la cote de $2 \mathrm{~m}$.

Cette observation, qui sera renouvelée en 1984, nous amènera à privilégier l'hypothèse d'un effet important de l'apport de matière organique sur le développement racinaire, et à étudier en détail ce facteur puisqu'aucune différence significative n'a pu être observée au niveau des caractéristiques hydrodynamiques du sol.

De plus, le suivi de l'évolution des profils hydriques montre que durant cette expérimentation interannuelle, 2 classes de problèmes sont rencontrées :

a) - des cas analogues à celui observé (fig. 2) en 1983 sur le traitement $\mathrm{T} 1$ où les profils hydriques en profondeur restent toujours à une teneur en eau faible (de l'ordre de $0,04 \mathrm{~cm}^{3} / \mathrm{cm}^{3}$ ) et identique à celle obtenue avant semis. Dans ces conditions, l'ordre de grandeur de la conductivité hydraulique est telle (autour de $0,1 \mathrm{~mm} /$ jour (fig. 1)) que l'on peut raisonnablement négliger tout transfert au-delà de la dernière cote de mesure. D'une façon classique l'évapotranspiration réelle peut alors être directement obtenue entre 2 dates par

$$
\mathrm{ETR}=\mathrm{P}+\Delta \mathrm{S}
$$

où $\mathrm{P}$ et $\Delta \mathrm{S}$ représentent la pluie et la variation totale $\mathrm{du}$ stock sur tout le profil $(0-3,7 \mathrm{~m})$, aucun ruissellement n'ayant pu être observé compte tenu de la nature

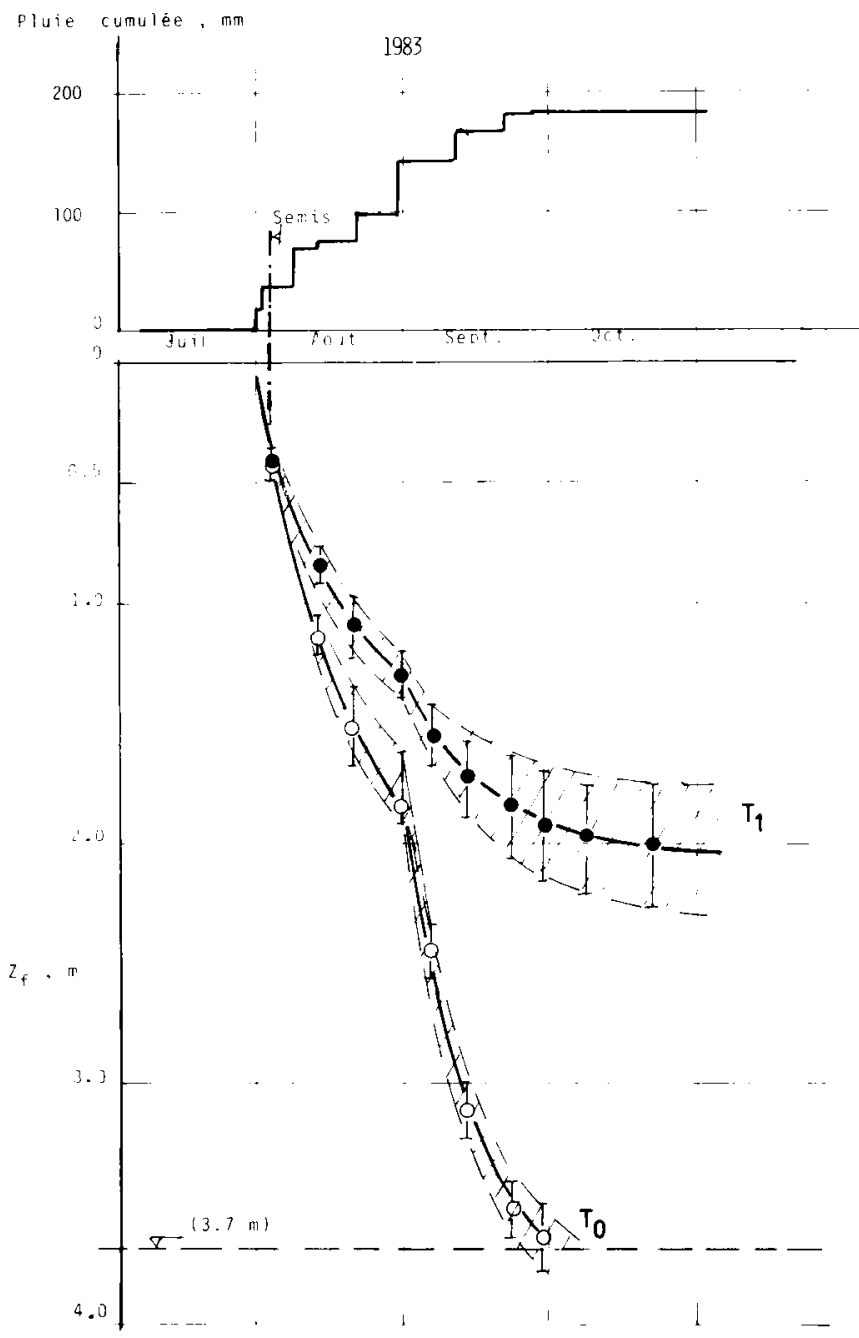

Figure 3

Evolution comparée de la cinétique de pénétration du front d'humidité dans le sol sous arachide en $1983 .\left(Z_{f}\right.$ profondeur $d u$ front d'humectation.)

Cumulative annual rainfall and penetration of the wetting front observed on Tl and TO in 1983 under groundnut. $\left(Z_{f}\right.$ depth of wetting front.) 
du sol. Les pertes en eau au-delà du front racinaire $Z_{r}$ - appelées encore drainage - seront alors calculées par :

$$
\operatorname{Dr}=\Delta S^{\prime}
$$

où $\Delta S^{\prime}$ représente la variation de stock entre $Z_{\mathrm{r}}(1,5 \mathrm{~m}$ sur arachide, $1,8 \mathrm{~m}$ sur mil) et la dernière cote de mesure.

b) - à l'inverse, des cas où des percolations ont manifestement lieu à travers la dernière cote de mesure, comme en 1983 sur T0 (fig. 3). Afin d'effectuer le bilan hydrique, on est alors amené à calculer le drainage au-delà du front racinaire en appliquant la loi de Darcy :

$$
\operatorname{Dr}=-K(\theta) \operatorname{grad} H
$$

où $K(\theta)$ et grad $H$ sont respectivement déduits de la relation reportée figure 1 , et obtenus par mesures tensiométriques (en 1984 et 1985). L'ETR entre 2 dates vaut alors :

$$
\mathrm{ETR}=\mathrm{P}+\Delta \mathrm{S}-\mathrm{Dr}
$$

Le calcul des stocks et des variations de stock, et le traitement des mesures d'humidité a été effectué par utilisation du logiciel AIDHYS mis au point à l'Institut de Mécanique de Grenoble (LATY et al., 1987).

\section{A. Aspect méthodologique : bilan sur arachide 1983}

A titre méthodologique, on considère d'abord dans un premier temps les résultats obtenus en 1983, où seule la série cultivée en arachide est, rappelons-le, instrumentée. Les profils hydriques typiques, donnés figure 2 , ont déjà fait ressortir le contraste entre les 2 traitements.

On trouvera figure 4 les courbes donnant l'évolution des variations de stock hydrique total $\Delta \mathrm{S}$ (mesuré entre 0 et $3,7 \mathrm{~m}$ ), avec comme référence l'état sec avant pluie de semis ainsi que la pluviométrie durant la période de culture. Deux conclusions s'imposent :

- très faible variabilité des mesures à l'intérieur d'un traitement ;

- mauvaise utilisation de l'eau sur le témoin, car malgré la faible pluviométrie utile $(145 \mathrm{~mm}), 60 \mathrm{~mm}$ d'eau non utilisée par la plante restent disponibles dans le sol à la récolte sur $\mathrm{T} 0$ alors que sur le traitement avec fumure organique toute la pluie est utilisée.

Le calcul du bilan de consommation, selon les méthodes explicitées préalablement et sur la base de mesures en général hebdomadaires, conduit aux résultats reportés tableau 3 . Ces valeurs concernent chaque fois 5 répétitions par traitement.
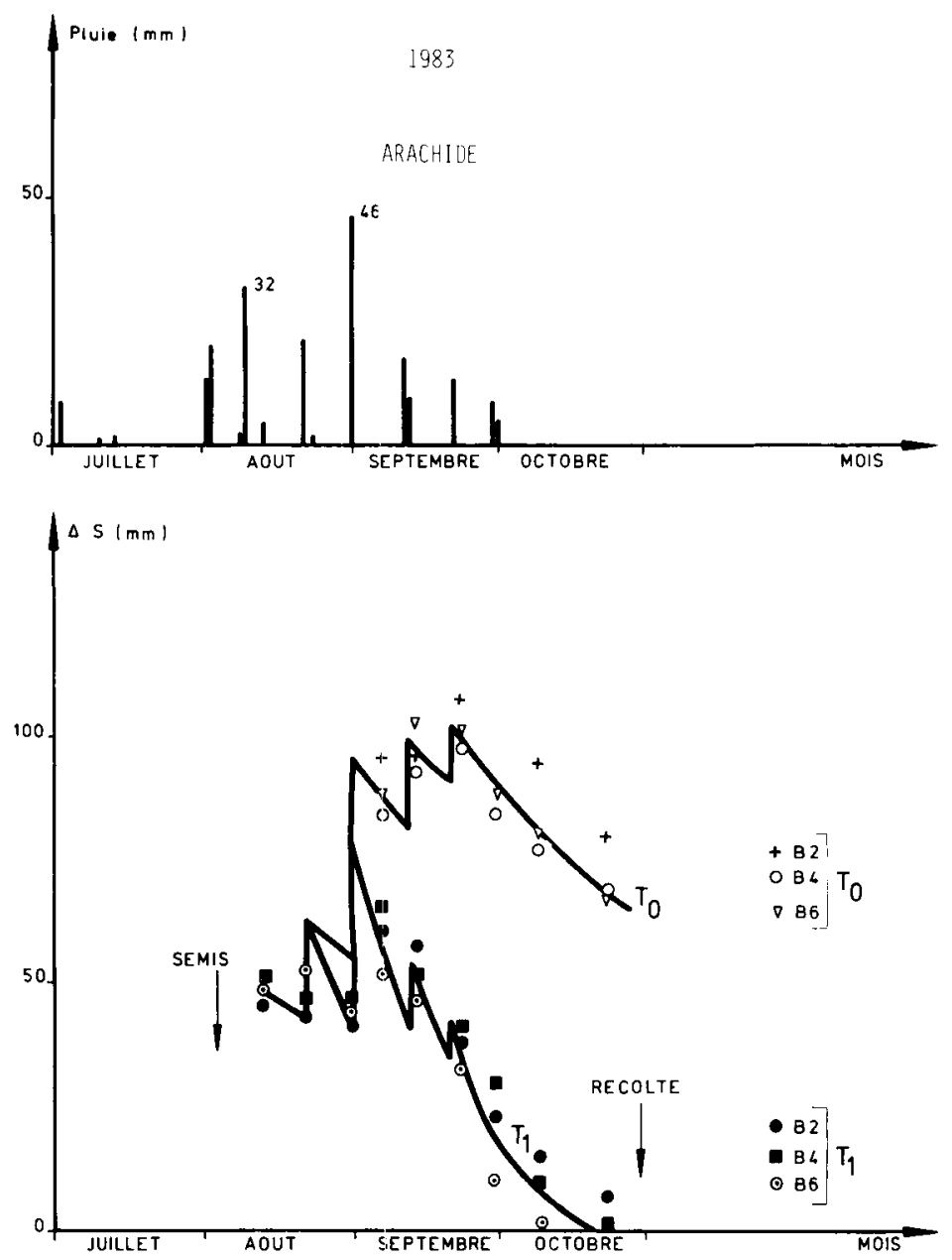
TABLEAU 3

Consommations hydriques journalières (mm/j), taux de satisfaction ETR/ETM (\%) des besoins en eau de l'arachide et drainage (mm) (3 répétitions par traitement); semis : 3/8/1983 - récolte : 29/10/1983 - pluie utile $145 \mathrm{~mm}$.

Evapotranspiration rate (ETR) ratio between water consumption and water requirement (ETR/ETM) and drainage below the root zone (Dr) during the crop cycle for treatment TO, T1, groundnut 1983. Sowing Aug. 3, harvesting Oct. 29, rain during the cycle $145 \mathrm{~mm}$ (3 replicates for each treatment).

\begin{tabular}{|c|c|c|c|c|c|c|c|}
\hline \multirow{3}{*}{$\begin{array}{l}\text { Période } \\
\text { (JAS) }\end{array}$} & \multirow{3}{*}{$\underset{(\mathrm{mm} / \mathrm{j})}{\mathrm{ETM}}$} & \multicolumn{3}{|c|}{ T0 } & \multicolumn{3}{|c|}{$\mathrm{T} 1$} \\
\hline & & \multirow{2}{*}{$\operatorname{ETR}(\mathrm{mm} / \mathrm{j})$} & ETR & \multirow{2}{*}{ Dr $(\mathrm{mm})$} & \multirow{2}{*}{$\operatorname{ETR}(\mathrm{mm} / \mathrm{j})$} & ETR & \multirow{2}{*}{ Dr $(\mathrm{mm})$} \\
\hline & & & ETM & & & ETM & \\
\hline $0-10$ & 3,32 & $2,10 \pm 0,75$ & 63 & - & $2,02 \pm 0,30$ & 61 & - \\
\hline $10-17$ & 3,31 & $0,91 \pm 0,11$ & 27 & $3,3 \pm 0,7$ & $1,09 \pm 0,07$ & 33 & - \\
\hline $17-27$ & 4,22 & $0,91 \pm 0,11$ & 22 & $8,7 \pm 1,9$ & $2,33 \pm 0,02$ & 55 & - \\
\hline $34-41$ & 5,81 & $2,15 \pm 1,09$ & 37 & $11,3 \pm 3,6$ & $4,20 \pm 0,46$ & 72 & $3,8 \pm 2,9$ \\
\hline $41-50$ & 6,26 & $0,71 \pm 0,90$ & 11 & $7,6 \pm 4,6$ & $3,54 \pm 0,45$ & 57 & $2,1 \pm 2,6$ \\
\hline $50-57$ & 5,02 & $3,40 \pm 0,35$ & 68 & $4,0 \pm 1,5$ & $2,80 \pm 0,57$ & 56 & $0,6 \pm 0,7$ \\
\hline $57-65$ & 4,66 & $1,30 \pm 0,43$ & 28 & $4,2 \pm 0,7$ & $1,07 \pm 0,21$ & 23 & - \\
\hline $65-79$ & 5,32 & $1,30 \pm 0,43$ & 24 & $4,0 \pm 0,1$ & $0, \dot{4} 1 \pm 0,16$ & 8 & - \\
\hline Cycle & $\operatorname{ETM}(\mathrm{mm})$ & $\operatorname{ETR}(\mathrm{mm})$ & & D total & $\operatorname{ETR}(\mathrm{mm})$ & & $\mathrm{D}$ total \\
\hline (semis-récolte) & 384,3 & $125,8 \pm 16,9$ & 33 & $64,6 \pm 8,8$ & $173,7 \pm 4,2$ & 45 & $9,5 \pm 6,4$ \\
\hline
\end{tabular}

Ces résultats sont synthétisés figure 5 sous 2 formes de présentation :

- consommation moyenne hebdomadaire durant le cycle. On notera de très fortes différences du taux de consommation intervenant entre le $20^{\mathrm{e}}$ et le $50^{\mathrm{e}}$ jour après semis (de la floraison au début de formation des gousses), où la consommation sur $\mathrm{T} 1$ est très significativement supérieure à celle sur T0, alors que durant les 20 premiers jours les valeurs sont identiques. A la fin, le processus s'inverse. Ce point sera discuté plus tard ;
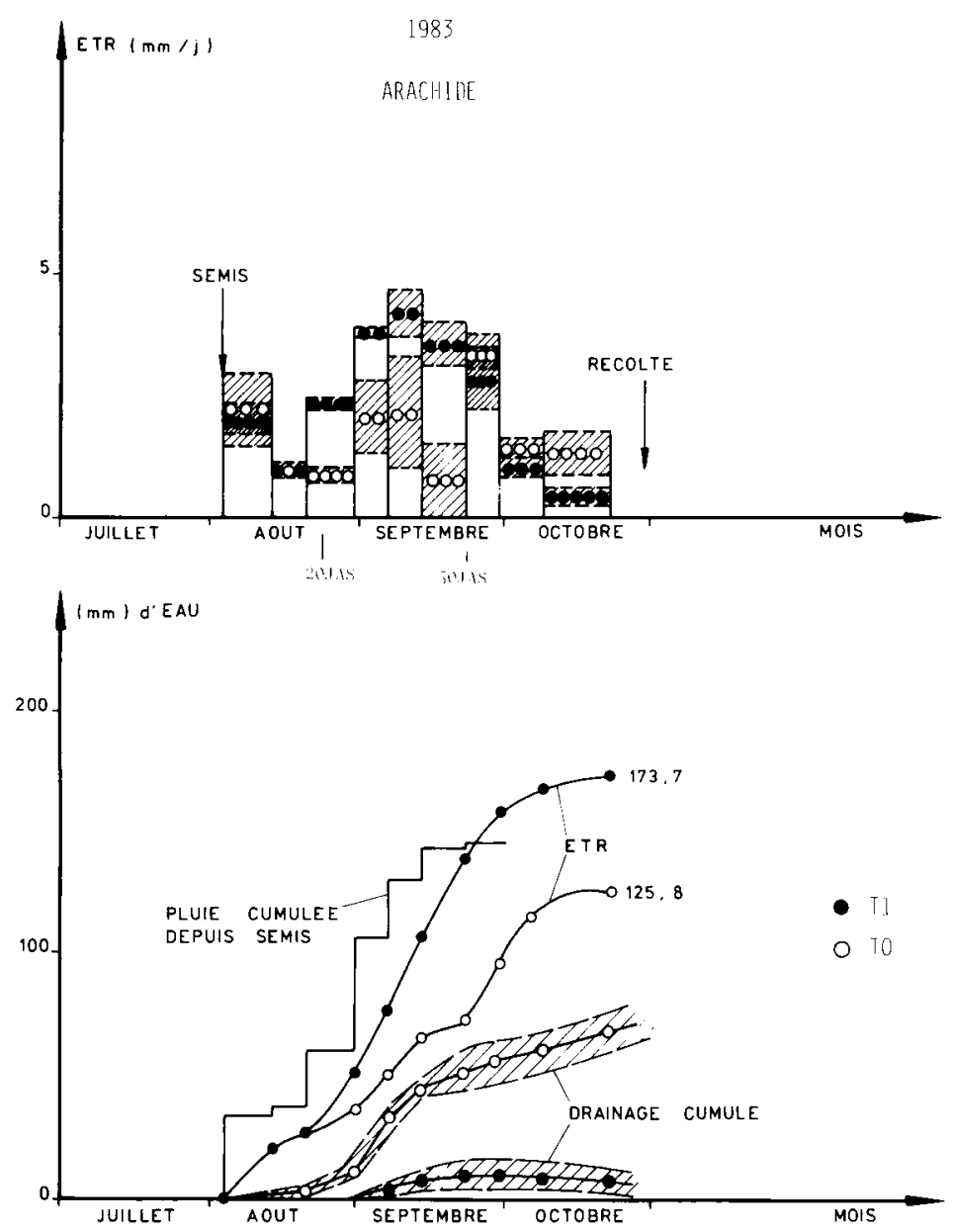

Figure 5

Arachide 1983 - Comparaison entre bilans cumulés et taux de consommation d'eau sur les 2 traitements. WIIt] Bandes d'incertitudes entre répétitions. $J A S=$ jours après semis.
Evapotranspiration rate, cumulative water consumption, cumulative drainage losses for groundnut on treatments Tl and T0, in 1983. WU Domain of uncertainty. JAS = days after sowing. 
- bilan cumulé : pluie totale utile, ETR et drainage. Dans le cas du traitement avec apport organique, la différence entre l'ETR totale $(174 \mathrm{~mm})$ et la pluie cumulée après semis représente l'utilisation du stock d'eau présent dans le sol juste avant le semis.

Sur la figure 5, les zones hachurées représentent les bandes d'incertitudes entre les 3 répétitions par traitement. Pour le calcul du drainage on a pour chaque site utilisé l'équation (3) avec la mesure locale de $\theta$ et de grad $H$ sur le tube à la cote de référence $(1,5 \mathrm{~m}$ pour l'arachide).

Dans tous les cas de figure, les taux de consommation sont très inférieurs 'aux besoins hydriques de la plante, comme le montre le rapport ETR/ETM, ce dernier terme étant calculé selon la méthode proposée par DANCETTE (1984). Enfin les pertes hydriques par drainage représentent respectivement 5 à 45 p. 100 de la pluviométrie utile sur $\mathrm{T} 1$ et $\mathrm{T} 0$, ce qui suggère une très mauvaise colonisation du sol par l'enracinement sur le traitement T0.

Au niveau des bilans de production, on obtient pour cette série de mesures respectivement $550 \pm 200$ et $1738 \pm 108 \mathrm{~kg}$ de matière sèche par hectare sur T0 et T1 (les détails de production sont donnés par CISSE, 1986). Clairement l'efficience est 2 fois plus élevée sur le traitement avec amendement organique. Ce point sera repris dans la discussion.

\section{B. Synthèse des résultats en 1984 et 1985}

Les 2 années suivantes sont fortement contrastées, puisque la pluviométrie utile obtenue en 1985 (302 $\mathrm{mm}$ ) est près de 50 p. 100 supérieure à celle caractérisant 1984 (environ $210 \mathrm{~mm}$ ). Il en résulte des comportements différents entre variétés et traitements. Du point de vue hydrique, on notera ainsi que les profils hydriques mesurés au maximum du stock hydrique montrent qu'aucune humectation n'a lieu en 1984 au-delà de $2 \mathrm{~m}$ alors qu'en 1985, suite à de fortes pluies intervenues durant la $2^{\mathrm{e}}$ quinzaine d'août $(160 \mathrm{~mm})$, d'importantes percolations traversent la cote $3,7 \mathrm{~m}$ avec une forte augmentation de la teneur en eau jusqu'à ce niveau.

Rappelons que pour ces 2 années on dispose chaque fois de 3 répétitions pour chacun de 2 traitements, sur mil et arachide (en rotation). Le détail des analyses est présenté dans la thèse de L. CiSSE (1986). On trouvera figure 6 l'évolution du taux de satisfaction des besoins en eau pour ces 2 cultures. Les valeurs globales en terme de bilan hydrique et bilan de production et efficience sont reportées tableaux 4 et 5 .
TABLEAU 5

Production totale (matière sèche) et efficience sur les deux cultures et pendant la durée de l'essai.

Total dry matter production and water efficiency for crops and treatments analyzed in this paper.

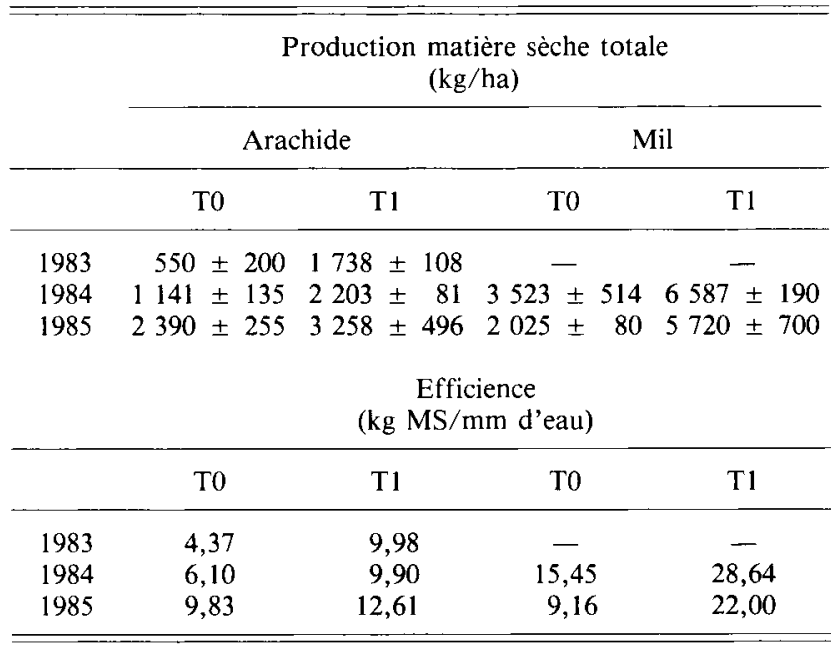

\section{Pour l'année 1984}

- pour le mil sur les 2 traitements, la consommation totale correspond à la pluie utile. Cependant des différences notables apparaissent au niveau des taux de consommation durant le cycle : identiques jusqu'au $20^{\mathrm{e}}$ jour après semis, on note une très forte augmentation sur le traitement $\mathrm{T} 1$ à la suite d'épisodes de pluie de $30 \mathrm{~mm}$ fin juillet et fin août (période de tallage début de formation des grains). En fin de cycle par contre le taux de consommation est significativement supérieur sur le témoin. Notons qu'aucun drainage n'a pu être mesuré au-delà de la cote $1,80 \mathrm{~m}$ sur l'ensemble des sites implantés sur cette culture.

- Pour l'arachide, comme en 1983, le traitement avec apport d'amendement organique a une consommation d'eau significativement supérieure à celle du traitement T0. Au cours du cycle, les taux moyens hebdomadaires se différencient à partir du $45^{\mathrm{e}}$ jour, pour rester sur Tl systématiquement supérieurs et à des valeurs voisines de l'optimum jusqu'au $80^{\mathrm{e}}$ jour. Audelà, le comportement s'inverse, avec un taux de consommation légèrement supérieur sur T0. Enfin le drainage au-delà de $1,5 \mathrm{~m}$ n'est mesurable que sur le traitement $T 0$, avec une valeur cumulée atteignant à la récolte $25 \pm 6 \mathrm{~mm}$ soit $10 \mathrm{p}$. 100 de la consommation totale.

TABLEAU 4

Valeurs globales : plixie utile, consommation et drainage.

Annual values of rainfall, real evapotranspiration and drainage losses below the root zone measured in 1984 and 1985 for the two crops and the two treatments.

\begin{tabular}{|c|c|c|c|c|c|c|}
\hline & \multicolumn{3}{|c|}{1984} & \multicolumn{3}{|c|}{1985} \\
\hline & $\mathrm{Pu} \mathrm{mm}$ & ETR mm & Dr mm & $\mathrm{Pu} \mathrm{mm}$ & ETR mm & Dr mm \\
\hline Arachide $\mathrm{T} 0$ & 211,8 & $187 \pm 6$ & $25 \pm 7$ & 302 & $243 \pm 27$ & $64 \pm 17$ \\
\hline Arachide $\mathrm{T} 1$ & 211,8 & $223 \pm 3$ & - & 302 & $258 \pm 13$ & $50 \pm 18$ \\
\hline Mil T0 & 229,3 & $228 \pm 8$ & - & 301,3 & $221 \pm 14$ & $94 \pm 7$ \\
\hline Mil T1 & 229,3 & $230 \pm 3$ & - & 301,3 & $260 \pm 13$ & $42 \pm 9$ \\
\hline
\end{tabular}



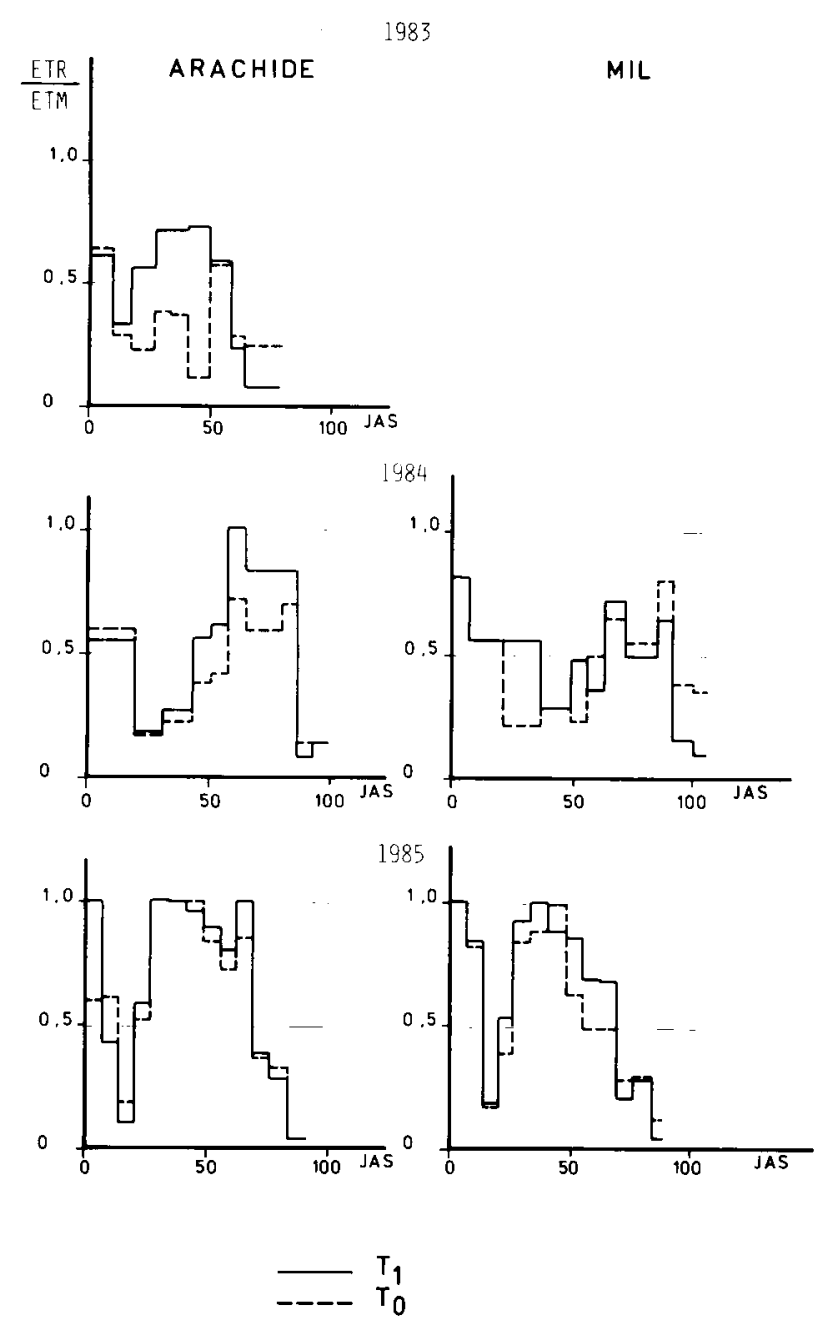

Figure 6

Evolution des taux de satisfaction des besoins de l'arachide et du mil durant l'expérimentation en 1983, 1984 et 1985. JAS = jours après semis.

Change in the ratio between water consumption (ETR) and water requirement (ETM) during the crop cycle for groundnut and millet after treatment in 1983, 1984 and 1985. JAS $=$ days after sowing.

On notera globalement que sur l'année le taux moyen de satisfaction des besoins est très faible, et voisin de 45 p. 100 pour les 2 cultures.

\section{Pour l'année 1985}

Les pertes d'eau (et d'éléments minéraux) affectent d'une façon importante les 2 cultures puisqu'après les fortes pluies $(160 \mathrm{~mm})$ intervenues dans la $2^{\mathrm{e}}$ quinzaine d'août, d'importantes percolations traversent la cote 3,7 $\mathrm{m}$ avec une forte augmentation de la teneur en eau. Leurs estimations, peut-être moins précises que dans les cas précédents puisque basées uniquement sur la loi de DARCY, atteignent les valeurs données tableau 4. On peut néanmoins avancer ces résultats :

- pour le mil, les différences, tant sur les valeurs cumulées (consommation, drainage) que sur les taux de consommation hebdomadaire sont très nettement marquées. L'étude détaillée faite par CISSE, 1986, montre que durant le cycle on a sur $\mathrm{T} 1$ une consommation supérieure après les fortes pluies de mi-août (30-40 jours après semis), puis durant la période florai- son - début de formation des grains (48-70 jours après semis), et une inversion en fin de cycle ;

- pour l'arachide par contre, on ne peut pas obtenir de différences significatives sur les valeurs cumulées, ni sur les taux de consommation dans le cycle.

Dans les 2 cas il reste à la récolte un stock résiduel de $60 \mathrm{~mm}$ environ par rapport à l'état initial avant pluie de semis. Globalement, en 1985, les besoins en eau sont mieux couverts qu'en 1984, surtout pour l'arachide $T 1$, si ce n'est en début de cycle.

\section{DISCUSSION ET CONCLUSION}

Les fortes différences observées au niveau des cinétiques d'humectation, des taux de consommation, de la production nous ont amenés à rechercher une explication par les différences de biomasse racinaire. Des mesures de densité racinaire ont été effectuées dans ce but à mi-cycle du développenıent racinaire du mil en 1984 et de l'arachide en 1985. Les profils correspondants sont donnés figure 7. En outre, en 1985, les mêmes mesures ont également été faites sur les 20 premiers $\mathrm{cm}$ du sol seulement, mais à différentes périodes.

Il est clair, pour le mil, que l'enracinement sur T1 est beaucoup plus dense en surface. Les contrastes se produisent assez tôt (à partir de la $2^{\mathrm{e}}$ semaine après la levée). Par contre en profondeur des observations visuelles sur fosses ont montré que le front racinaire descend au-delà de 1,7 m sur T0, alors qu'il s'arrêtait à $1,4 \mathrm{~m}$ sur $\mathrm{T} 1$.

Pour l'arachide, le contraste est moins important en terme de masse, mais les racines s'étalent latéralement à une plus grande distance du pivot sur le traitement T1 (jusqu'à $0,4 \mathrm{~m}$ dans l'interligne au lieu de $0,15 \mathrm{~m}$ pour T0); elles présentent en outre une densité de nodules plus nombreuses.

Il est indéniable que l'apport de matière organique en surface favorise en début de cycle le développement racinaire. Ceci induit un double effet $=$ meilleure utilisation de l'eau du sol et développement accru de la plante sur T1. A l'inverse, en fin de cycle, et comme le montre clairement une autre étude publiée par ailleurs (CISSE \& VACHAUD, 1988b), l'accumulation de l'eau en profondeur sur T0 résultant d'une mauvaise efficience du système racinaire en surface induirait un développement et une extraction racinaire au-delà de $1 \mathrm{~m}$. Ceci expliquerait la tendance observée systématiquement d'une augmentation du taux de consommation sur $\mathrm{T} 0$ en fin de cycle par rapport à $\mathrm{T} 1$ où de telles réserves n'existent généralement pas.

Enfin l'analyse des résultats en termes de relation consommation-production, globalisés sur le cycle de culture, conduit à la figure 8 , dérivée du tableau 5 . Pour chaque année et chaque traitement, les valeurs obtenues sur chaque bloc sous forme «production de matière sèche totale-ETR durant le cycle " sont inscrites dans un quadrilatère caractérisant la zone d'incertitude à la réponse. On trouve aussi les valeurs résultant de la campagne 1986 (pluviométrie utile $204 \mathrm{~mm}$ ) lors d'un suivi plus léger n'ayant pas permis l'analyse détaillée faite préalablement. Deux résultats marquants nous semblent devoir être signalés.

En premier lieu, la réaction des 2 cultures à l'amendement organique apparaît très différente. Pour l'ara- 
a

MI-CYCLE

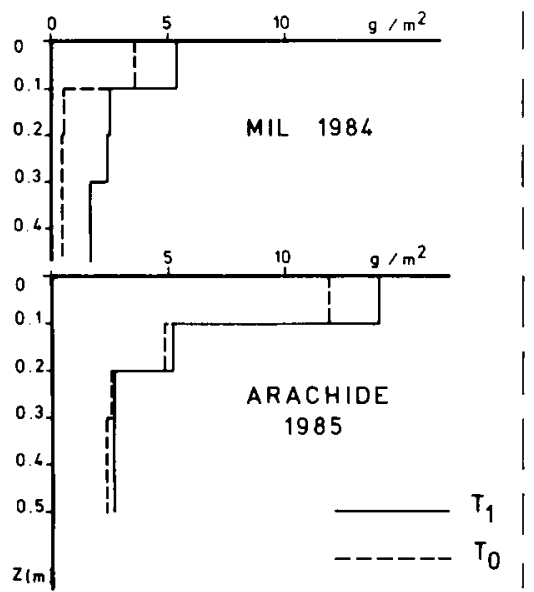

b

I VOLUTION DANS LE CYCIE

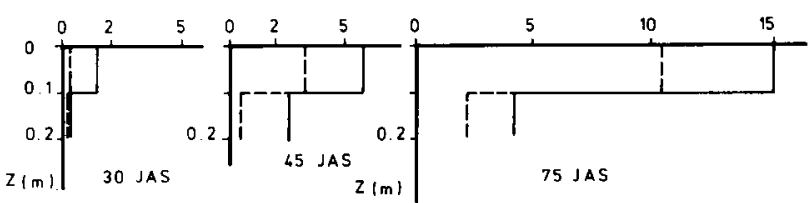

MIL 1985

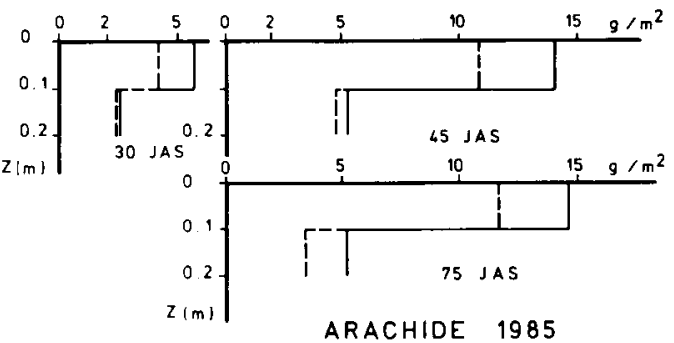

Figure 7

a) Profil de densité racinaire $\left(\mathrm{g} / \mathrm{m}^{2}\right)$ à mi-cycle (1984 sur mil, 1985 sur arachidej et b) évolution de la densité racinaire à différentes périodes en 1985 dans les tranches 0-0,1 et 0,1-0,2 m.JAS = jours après semis.

a) Root density $\left(\mathrm{g} / \mathrm{m}^{2}\right)$ profiles measured 45 days after germination for millet in 1984 and groundnut in 1985 on TI and TO; b) change in root density in the surface horizons $(0-0.2 \mathrm{~m})$ during the crop cycle for groundnut and millet in 1985. JAS = days after sowing.

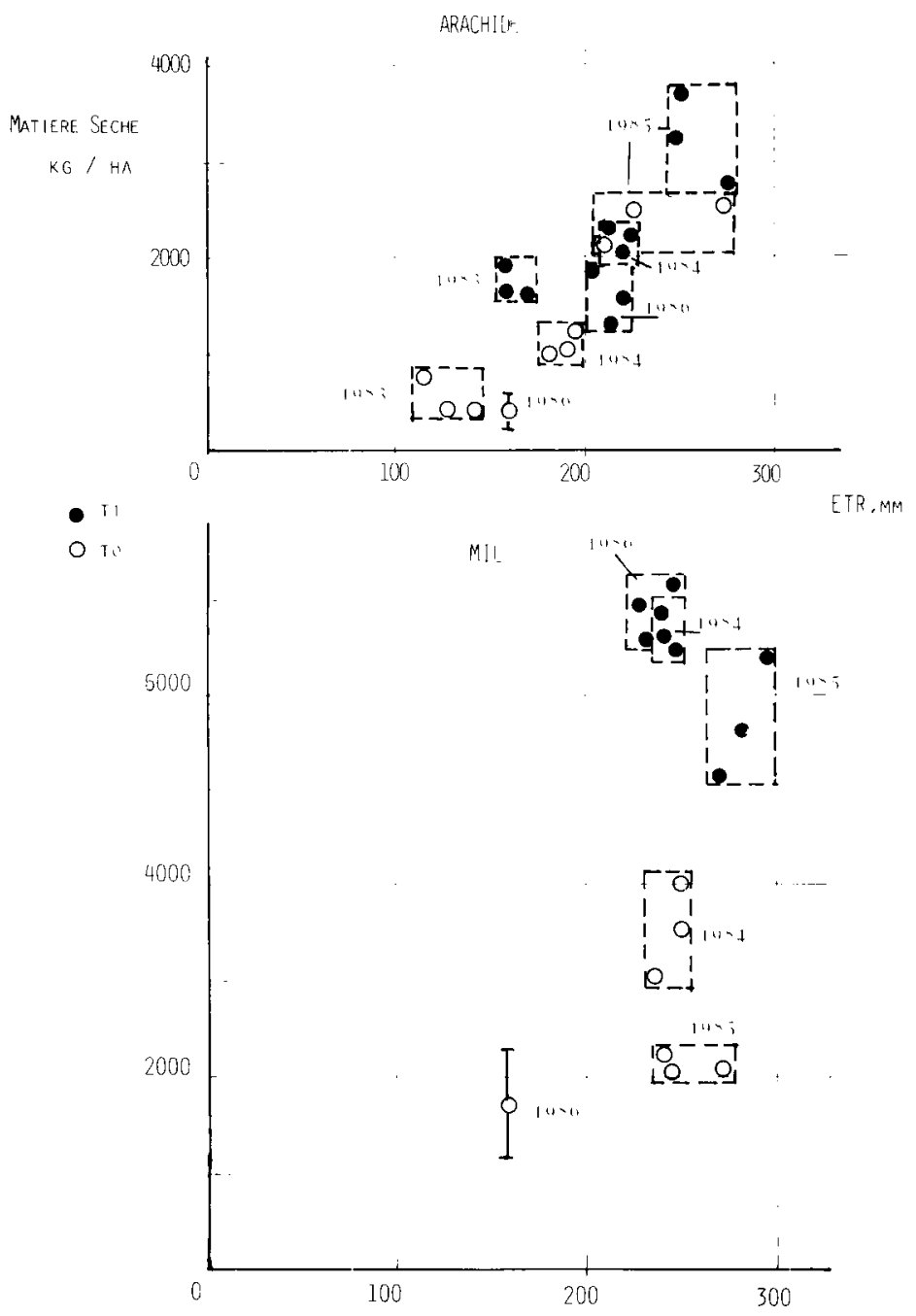

Figure 8

Liaison entre production de matière sèche totale et consommation.

Relation between total dry matter production and total water con sumption in relation 10 crop and treatment. 
chide les valeurs de rendement total semblent peu affectées par le niveau de fertilité, mais surtout par l'alimentation hydrique, avec un accroissement de $2 \mathrm{~T} /$ ha par $100 \mathrm{~mm}$ annuel d'ETR. Pour le mil au contraire, le niveau de fertilité semble avoir une importance essentielle et l'alimentation hydrique n'est pas un paramètre très explicatif du rendement.

Ces résultats doivent néanmoins être affinés. Il s'agit ici d'expérimentation en milieu extrême (faible pluviométrie, sol très filtrant) sur une durée insuffisante pour effectuer une analyse plus fine en fonction des phases de développement (DANCETTE, 1984). Ils nous semblent néanmoins pouvoir être mis à profit, notamment à un moment où l'utilisation de modèle de prévision de rendement (FOREST \& KALMS, 1984) s'avère être un outil indispensable d'aide à la décision, pour inciter à leur amélioration en s'attachant à 2 aspects que nous considérons comme essentiels, et liés :

- la zone racinaire, puisque le paramètre considéré figure 8 est l'ETR et non la pluie totale, tion.

- la réaction de la plante au niveau de la fertilisa-

En second lieu, l'efficience des cultures est remarquable. Il faut certes affiner les résultats en tenant compte, comme le fait L. CISSE (1986) de la produc- tion de grains ou de gousses d'une part, et de pailles ou fanes d'autre part. Néanmoins des rendements de 6 à 7 tonnes par hectare de matière sèche pour une consommation de $200 \mathrm{~mm}$ d'eau sont obtenus sur le mil. L'apport de matière organique conduit globalement à une production 2 à 3 fois plus forte que sur le témoin.

Il est clair que la dose de fumier utilisée dans cette expérimentation est très supérieure à celle que les disponibilités du milieu étudié permettent d'appliquer. Une nouvelle expérimentation est actuellement en cours dans des conditions de milieu analogue pour déterminer à la fois les doses minimum conduisant à un effet significatif, et le temps nécessaire pour obtenir ces effets.

Reç le 18 juin 1987. Accepté le 19 janvier 1988.

\section{REMERCIEMENTS}

Cette étude a été effectuée dans le cadre d'une convention d'Assistance Technique entre l'Agence Internationale pour l'Energie Atomique et l'Institut Sénégalais de Recherche Agricole. La contribution de l'AIEA en fourniture d'équipement, missions d'experts et bourses de formation a été essentielle.

Les auteurs tiennent en outre à associer à ce travail Mr A. FAYE, M. Goudiaby et M. Thiaw, techniciens du CNRA Bambey, à qui est due la qualité des mesures.

\section{RÉFÉRENCES BIBLIOGRAPHIQUES}

Bonfils P., Faure J., 1959. Les sols de la région des Thies. Bull. Agron. ORSTOM, 16, 5-92.

Cisse L., 1986. Etude des effets d'apports de matière organique sur les bilans hydriques et minéraux et la production de mil et d'arachide sur un sol sableux dégradé du Centre-Nord du Sénégal. Thèse Dr. Ing., Inst. Nat. Polyt. de Lorraine, 184 p., nov. 1986.

Cisse L., 1988. Influence d'apports de matière organique sur la culture de mil et d'arachide sur un sol sableux du Nord-Sénégal. II Développement des plantes et mobilisations minérales. Agronomie, 8 (5).

Cisse L., Vachaud G., 1988a. Etude de l'influence de méthodes d'amendement sur l'infiltration, l'évaporation en début de saison des pluies et les coefficients transferts hydriques d'un sable dégradé du Nord Sénégal. Revue d'Hydrologie Continentale, ORSTOM, vol. II, $n^{\circ} 1,15-28$.

Cisse L., Vachaud G., 1988b. Caractérisation de l'extraction racinaire par tranche de sol par analyse de profils d'humidité. Influence des traitements sur la consommation en eau de mil et d'arachide. Agron. trop. Paris (sous presse).

Chopart J. L., 1980. Etude au champ des systèmes racinaires des principales cultures pluviales du Sénégal. Thèse de Doctorat d'Université, Toulouse, 159 p. +45 p. ann.
Dancette C., 1974. Les besoins en eau des plantes de grande culture au Sénégal, in Symposium "Use of radioisotopes and radiations techniques in Soil Physics and Irrigation Studies", Vienne, Autriche, 1973, AIEA SM 176/36, p. 351-372.

Dancette C., 1984. Agroclimatologie et adaptation variétale de l'arachide au Sénégal. Doc. Mult. CNRA/Bambey.

Forest F., Kalms J. M., 1984. Influence du régime d'alimentation en eau sur la production de riz pluvial. Simulation du bilan hydrique. Agron. trop. Paris, 39, 1, 42-50.

Imbernon J., 1982. Variabilité spatiale des caractéristiques hydrodynamiques d'un sol du Sénégal. Thèse Doctorat de $3^{\mathrm{e}}$ cycle, Univ. Scientifique et Médicale de Grenoble, 152 p.

Laty R., Vachaud G., 1987. AIDHYS, logiciel d'aide au stockage et au traitement de mesure hydriques dans le sol. Rapport interne, Institut de Mécanique de Grenoble, $33 \mathrm{p}$.

Pieri C., 1976. L'acidification des terres de culture exondées au Sénégal. Agron. trop. Paris, 21, 4, 339-368.

Vauclin M., Haverkamp R., Vachaud G., 1983. Analyse des erreurs liées à l'utilisation de l'humidimètre neutronique, in Colloque sur "l'Utilisation des ravonnements et des isotopes en Physique du Sol et en Irrigation » Aix-en-Provence, Ed. Agence Int. Energie Atomique, Vienne, p. 533-549. 